Supporting Information for

\title{
Rad6/Rad18 competes with DNA polymerases $\eta$ and $\delta$ for PCNA encircling DNA
}

\author{
Mingjie Li†, Leah Larsen ${ }^{\dagger}$, and Mark Hedglin ${ }^{\dagger}, *$ \\ Department of Chemistry, The Pennsylvania State University, University Park, PA 16802, \\ U.S.A. \\ *Author to whom correspondence should be addressed, Email: muh218@psu.edu
}

This PDF file includes the following:

Figures S1 to S6

\section{Supporting Figures and Legends}

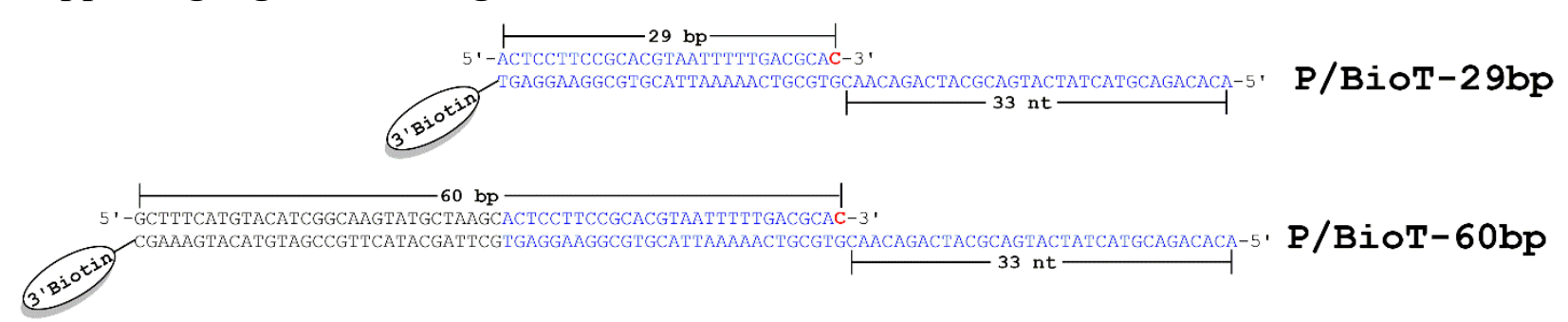

Figure S1 The P/T DNA substrates utilized in this study. The double-stranded $\mathrm{P} / \mathrm{T}$ regions are sufficient for assembly of a PCNA clamp onto DNA by the human clamp loader, replication factor $\mathrm{C}$ (RFC). The ssDNA regions (33 nt) adjacent to the 3 ' end of the P/T junction is consistent with the footprint of a RPA heterotrimer complex $(30 \pm 2 \mathrm{nt})$ at physiological ionic strength $(200 \mathrm{mM})$. RPA prevents loaded PCNA from sliding off the ssDNA end of the substrate. When pre-bound to Neutravidin, the biotin attached to the 3 '-end of the template strand prevents loaded PCNA from sliding off the dsDNA, i.e. duplex, end of the substrate. The 3' end of the primer is terminated with a dideoxy cytosine nucleotide (denoted by $\mathrm{C}$ ) that cannot be extended. The DNA substrates are identical (highlighted in blue) except the duplex region of P/BioT-60bp DNA is extended by $31 \mathrm{bp}$. 

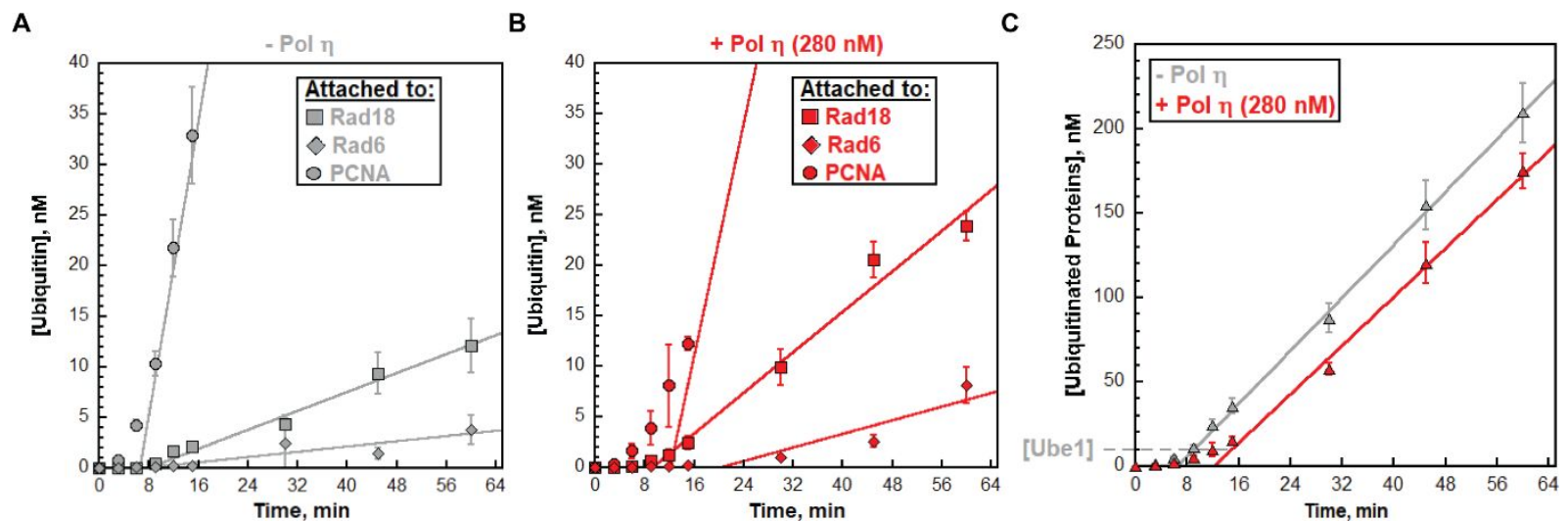

Figure S2. Biphasic behavior is observed for all ubiquitination events. Data points for each condition represent the average \pm S.D. of three independent experiments. $(\mathbf{A}-\mathbf{B})$ The concentration of ubiquitin attached to each target protein (Rad18, PCNA, Rad6) is plotted as a function of time for reactions carried out in the absence (panel A) or presence of pol $\eta$ (panel $\mathbf{B}$, $280 \mathrm{nM}$ ). Symbols for each target protein are indicated in the respective figure legend. For each target protein, data points from $t=15$ to $t=60 \mathrm{~min}$ are fit to linear regressions. The $y$-axes are limited to highlight Rad18 and Rad6. All data for PCNA is depicted in Figure 2B in the main text. (C) The total concentration of ubiquitinated target proteins from reactions depicted in panel $\mathbf{A}$ and $\mathbf{B}$ is plotted as a function of time. Symbols for each condition are indicated in the figure legend. Data points from $t=15$ to $t=60 \mathrm{~min}$ are fit to linear regressions. The ends of the lag phases are indicated by conformity to linearity and each coincides with the concentration of Ube 1 in the reactions ( $10 \mathrm{nM}$, indicated). 


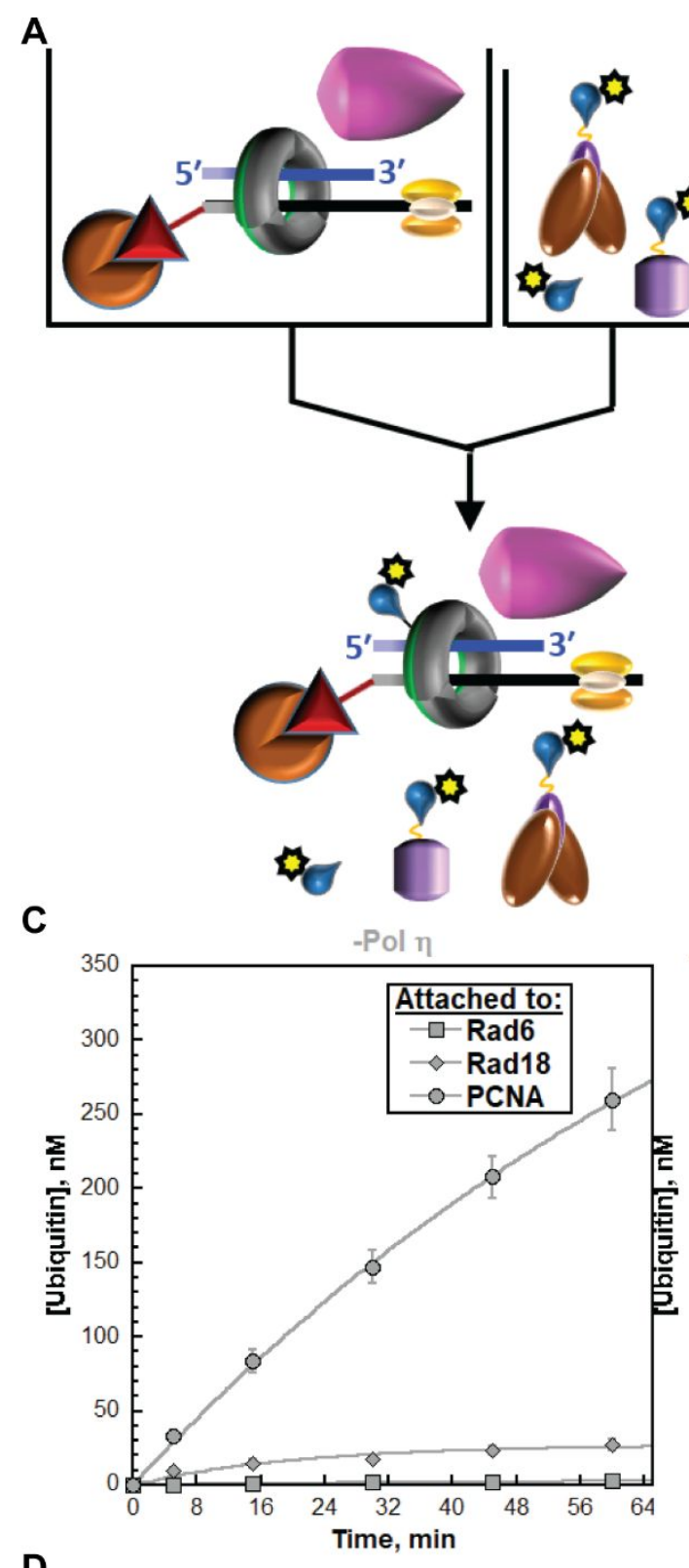

B

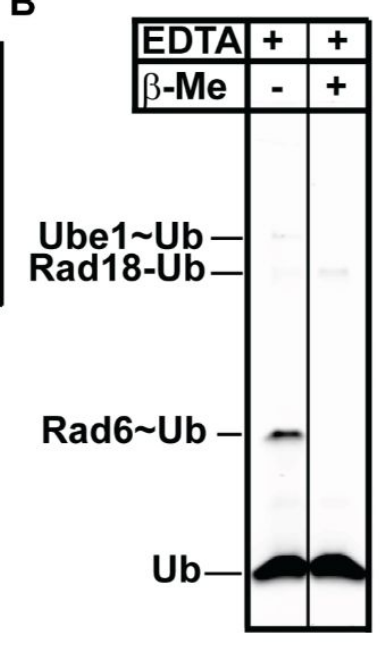

D
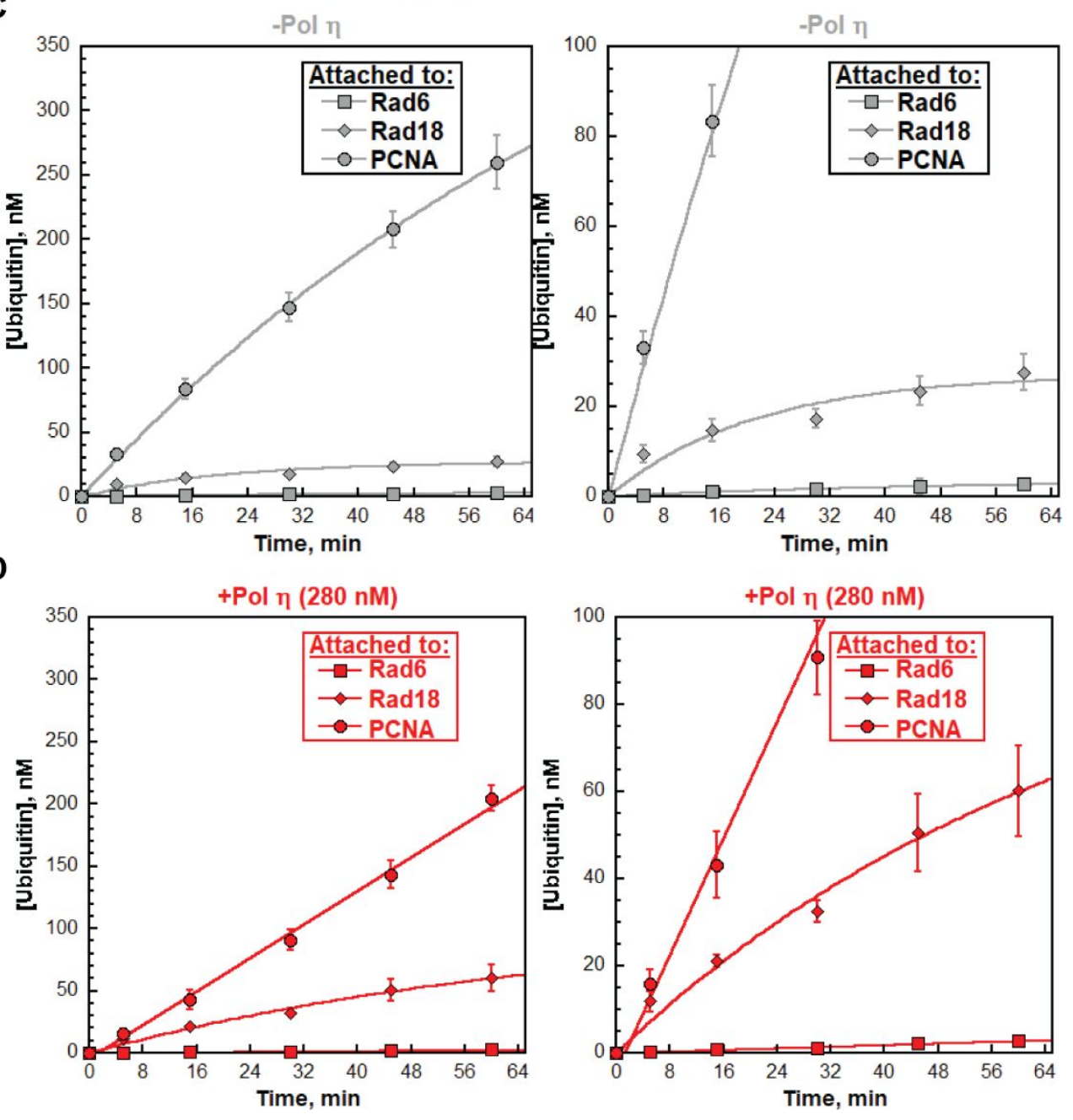
Figure S3 The lag phases represent charging of Rad6/Rad18 with ubiquitin. (A) Schematic representation of the experimental procedure. Scheme is identical to that described in Figure 2A in the main text except $\operatorname{Rad} 6 / \operatorname{Rad} 18(110 \mathrm{nM}$ heterotrimer) is pre-charged with ubiquitin (Fl-Ub, $1 \mu \mathrm{M})$ by Ube $1(10 \mathrm{nM})$ for 12 mins and ubiquitination is initiated by addition of this mixture. (B) Quantitative analysis of Rad6/Rad18 charging. Samples were removed from the Rad6/Rad18 pre-charging reaction and quenched after a 12 min incubation with either $1 \mathrm{X}$ non-reducing loading buffer ( $\beta-\mathrm{Me}$, lane 1$)$ or $1 \mathrm{X}$ reducing loading buffer $(+\beta-\mathrm{Me}$, Lane 2$)$. To ensure efficient quenching in the absence of $\beta$-Me, both quench solutions were supplemented with 188 mM EDTA (final concentration). Both thioester (represented by “ $~ ")$ and isopeptide bonds (represented by "_") connecting ubiquitin to proteins are maintained under non-reducing conditions. Only the latter are maintained under reducing conditions. As expected, ubiquitincharged Ube1 (Ube1 Ub) and ubiquitin-charged Rad6 (Rad6 Ub) are only observed under nonreducing conditions. At 12 mins, nearly all Rad6 $(94.5 \pm 18.7 \%)$ is pre-charged with ubiquitin. (C - D) Ubiquitination of target proteins. Data points for each condition represent the average \pm S.D. of three independent experiments. The concentration of ubiquitin attached to each target protein $(\operatorname{Rad} 18$, PCNA, Rad6) are plotted as a function of time for reactions carried out in the absence (panel C) or presence of pol $\eta$ (panel D, $280 \mathrm{nM}$ ). All data for a given condition is shown in the left graph of the respective panel whereas the y-axes are limited in the right graph of the respective panel to highlight Rad18 and Rad6. Under these conditions where nearly all Rad6/Rad18 is pre-charged with ubiquitin prior to the start of the reaction, lag phases are absent for each target protein. This contrasts Figure S2 and Figure 2B in the main text where significant lag phases are observed when $\operatorname{Rad6} / \operatorname{Rad} 18$ is uncharged at the onset of the reaction. Together, this suggests that the lag phases represent charging of Rad6/Rad18 with ubiquitin. Under the conditions described in panel $\mathbf{A}$, monoubiquitinated PCNA appears to increase in a linear manner in the presence of $280 \mathrm{nM}$ pol $\eta$ but increases in an exponential manner in the absence of pol $\eta$. Hence, the extent of inhibition cannot be calculated from these divergent behaviors. 

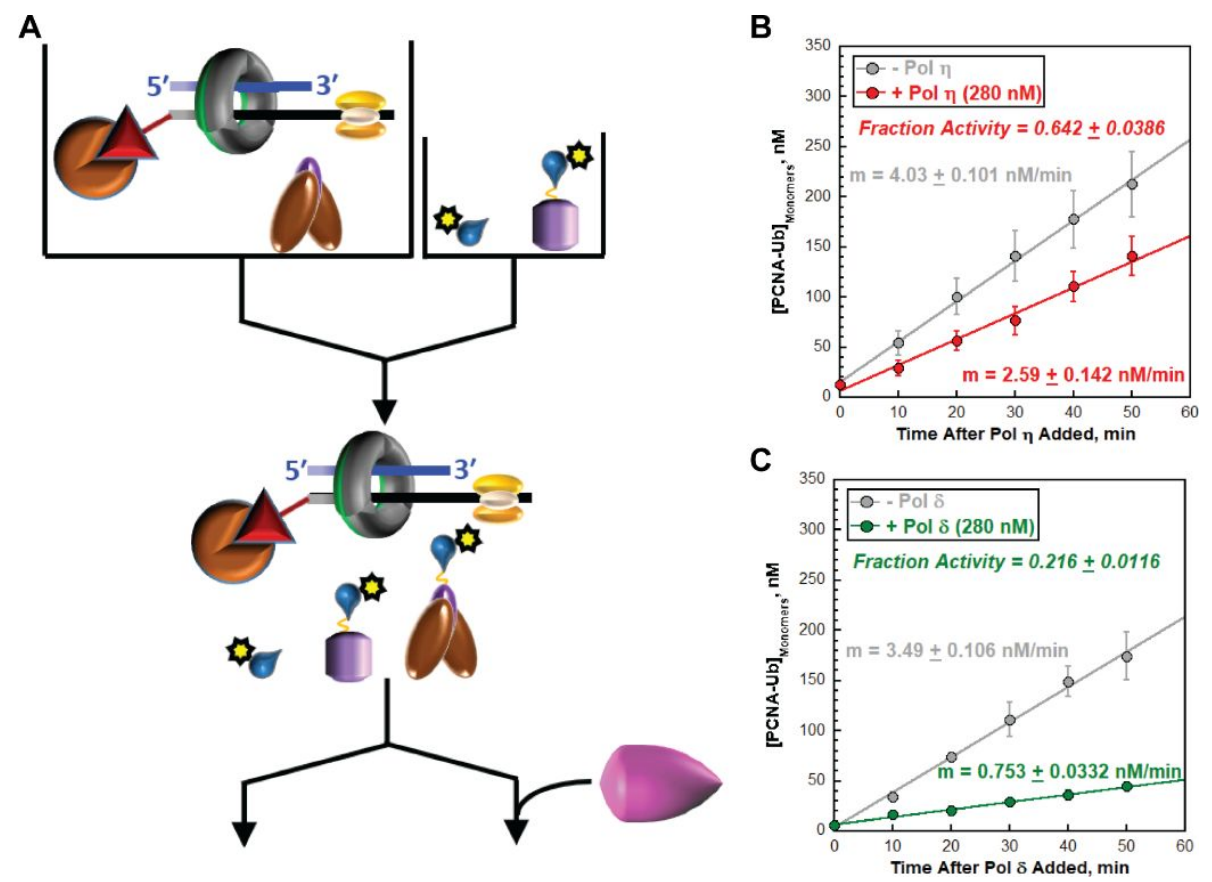

Figure S4 Pols $\eta$ and $\delta$ inhibit transfer of ubiquitin from Rad6/Rad18 to PCNA. (A) Schematic representation of the experimental procedure. The P/BioT-29bp DNA substrate (110 nM with $440 \mathrm{nM}$ Neutravidin) is saturated with RPA (110 nM) and PCNA (110 nM homotrimer) is preloaded by RFC (110 nM). Uncharged Rad6/Rad18 (110 nM heterotrimer) is added and then ubiquitination of target proteins is initiated by addition of a pre-incubated mixture of fluorescently-labeled ubiquitin (F1-Ub, $1 \mu \mathrm{M})$ and Ube1 $(10 \mathrm{nM})$. The reaction is divided into equal volume aliquots. The average lag time for all control reactions (i.e., "- pol") in the present study is $4.53 \pm 2.56 \mathrm{~min}$. After $5.0 \mathrm{~min}$, a pol is added to one aliquot and pol storage buffer is added to the other. Covalent attachment of ubiquitin to all target proteins is monitored over time for each aliquot. (B - C) Quantitative analysis of PCNA monoubiquitination. Data points for each condition represent the average \pm S.D. of three independent experiments. The concentration of monoubiquitinated PCNA monomers observed in the absence and presence of a pol is plotted as a function of the elapsed time after addition of the pol. Data for pol $\eta(280 \mathrm{nM})$ and pol $\delta(230$ $\mathrm{nM}$ ) is shown in panels $\mathbf{B}$ and $\mathbf{C}$, respectively. Symbols for each condition are indicated. For all conditions, a lag phase is absent and, hence, all data points are fit to a linear regression. Standard errors of the slopes $(\mathrm{m})$ are reported. From the slope values, the fraction activities (indicated) are calculated. 
A

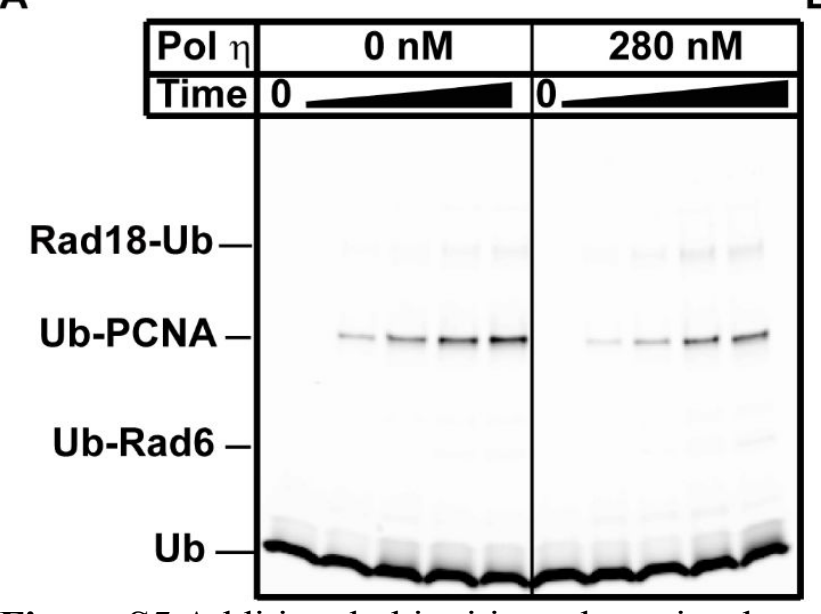

B

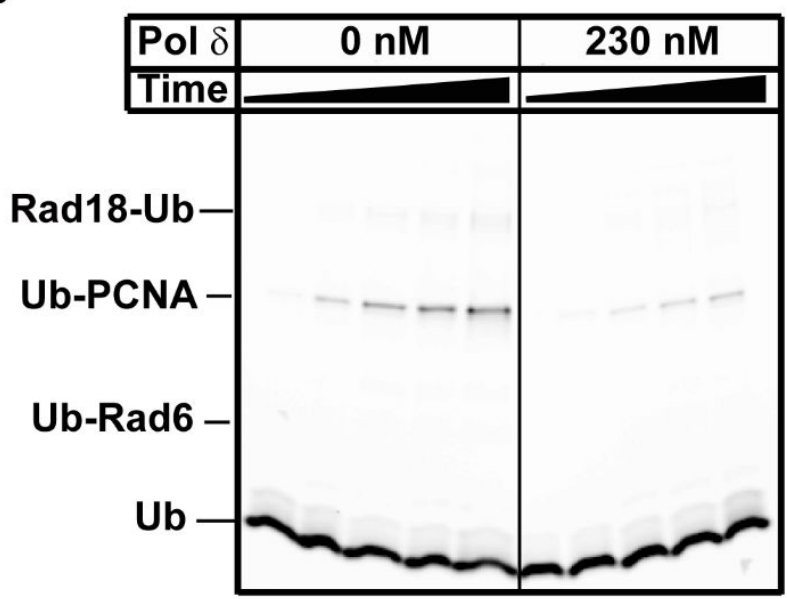

Figure S5 Additional ubiquitinated species do not appear in the presence of pols. Fluorescence scan of reactions carried out in the absence and presence of a pol are shown. Ubiquitin ( $\mathrm{Ub})$, monoubiquitinated PCNA (Ub-PCNA), monoubiquitinated Rad6 (Ub-Rad6), and ubiquitinated $\operatorname{Rad} 18\left(\operatorname{Rad} 18-(\mathrm{Ub})_{\mathrm{n}}\right)$ are indicated. Shown in panels $\mathbf{A}$ and $\mathbf{B}$ are images for pol $\eta( \pm 280 \mathrm{nM})$ and pol $\delta( \pm 230 \mathrm{nM})$, respectively.

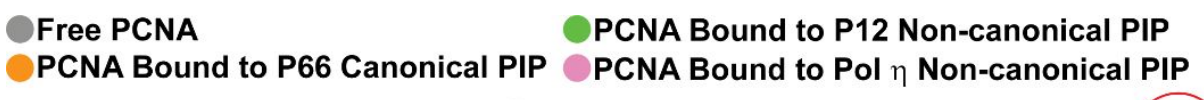

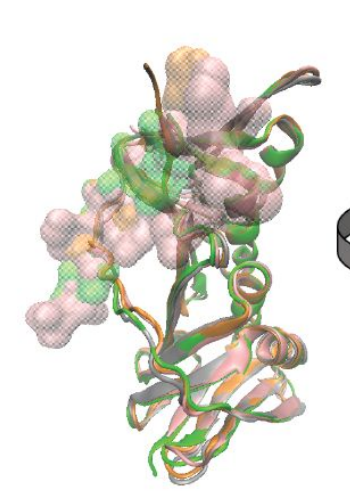

Front

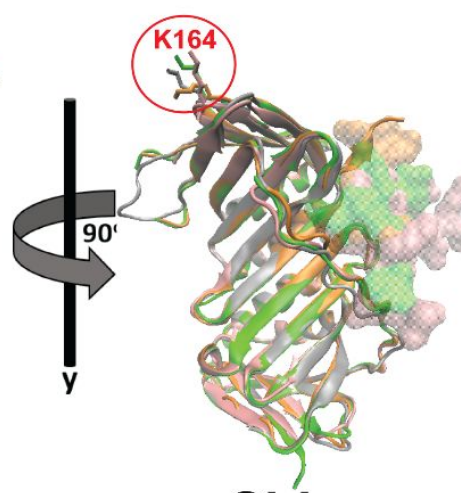

Side

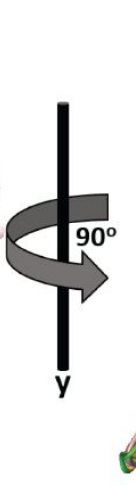

$7^{90^{\circ}}$

Back

Figure S6 Binding of the PCNA IDCL by PIP box motifs does not distort the PCNA structure. Crystal structures of PCNA clamps associated with multiple copies of either the canonical PIP box motif of the human pol $\delta$ P66 subunit (PDB code 1U76), the non-canonical PIP box motif of the human pol $\delta$ P12 subunit (PDB code 6HVO), or a non-canonical PIP motif of human pol $\eta$ (PDB code 2ZVK) have been reported ${ }^{l-3}$. These structures were aligned along the PCNA peptide backbones with unbound PCNA (PDB code 3JAP) using VMD ${ }^{4}$. For simplicity, only PCNA monomers are displayed. The coloring scheme is indicated. PCNA backbones are displayed in new cartoon form with K164 residues (indicated) shown in bond form. The PIP box motifs are shown as transparent surfaces. The RMSDs for the peptide backbones, amino acid residues 159 to 169 , and K164 residues are $\leq 0.83, \leq 1.29, \leq 2.25$, respectively. Altogether, this indicates that binding of the canonical and non-canonical PIP box motifs of pols $\delta$ and $\eta$ does not distort the structure of PCNA, the ubiquitin attachment site (K164) in particular. 


\section{Supporting References}

[1] Bruning, J. B., and Shamoo, Y. (2004) Structural and thermodynamic analysis of human PCNA with peptides derived from DNA polymerase-delta p66 subunit and flap endonuclease-1, Structure 12, 2209-2219.

[2] Gonzalez-Magana, A., de Opakua, A. I., Romano-Moreno, M., Murciano-Calles, J., Merino, N., Luque, I., Rojas, A. L., Onesti, S., Blanco, F. J., and De Biasio, A. (2019) The p12 subunit of human polymerase uses an atypical PIP box for molecular recognition of proliferating cell nuclear antigen (PCNA), Journal of Biological Chemistry 294, 3947-3956.

[3] Hishiki, A., Hashimoto, H., Hanafusa, T., Kamei, K., Ohashi, E., Shimizu, T., Ohmori, H., and Sato, M. (2009) Structural basis for novel interactions between human translesion synthesis polymerases and proliferating cell nuclear antigen, $J$ Biol Chem 284, 10552-10560.

[4] Lau, W. C., Li, Y., Zhang, Q., and Huen, M. S. (2015) Molecular architecture of the UbPCNA/Pol eta complex bound to DNA, Sci Rep 5, 15759, 1 - 11. 